\title{
Further Results about Traveling Wave Exact Solutions of the Drinfeld-Sokolov Equations
}

\author{
Fu Zhang, ${ }^{1}$ Jian-ming Qi, ${ }^{1}$ and Wen-jun Yuan ${ }^{2}$ \\ ${ }^{1}$ Department of Mathematics and Physics, Shanghai Dianji University, Shanghai 201306, China \\ ${ }^{2}$ School of Mathematics and Information Science, Guangzhou University, Guangzhou 510006, China \\ Correspondence should be addressed to Jian-ming Qi; qijianmingdaxia@163.com
}

Received 11 May 2013; Revised 11 September 2013; Accepted 17 September 2013

Academic Editor: Chein-Shan Liu

Copyright (c) $2013 \mathrm{Fu}$ Zhang et al. This is an open access article distributed under the Creative Commons Attribution License, which permits unrestricted use, distribution, and reproduction in any medium, provided the original work is properly cited.

We employ the complex method to obtain all meromorphic exact solutions of complex Drinfeld-Sokolov equations (DS system of equations). The idea introduced in this paper can be applied to other nonlinear evolution equations. Our results show that all constant and simply periodic traveling wave exact solutions of the equations (DS) are solitary wave solutions, the complex method is simpler than other methods and there exist simply periodic solutions $v_{s, 3}(z)$ which are not only new but also not degenerated successively by the elliptic function solutions. We believe that this method should play an important role for finding exact solutions in the mathematical physics. For these new traveling wave solutions, we give some computer simulations to illustrate our main results.

\section{Introduction}

In this work, we aim to further study the Drinfeld-Sokolov system (Drinfeld-Sokolov equations (DS system of equations)) [1-3]

$$
\begin{gathered}
u_{t}+\left(v^{2}\right)_{x}=0, \\
v_{t}-a v_{x x x}+3 d v u_{x}+3 k u v_{x}=0,
\end{gathered}
$$

where $a, d$, and $k$ are constants. This system was introduced by Drinfeld and Sokolov as an example of a system of nonlinear equations possessing Lax pairs of a special form [1].

Wazwaz [4] used the sine-cosine method and the tanh method to stress the power of these methods to nonlinear equations. Wazwaz [4] also investigated the traveling wave solutions with compact and noncompact structures for the Drinfeld-Sokolov equations (DS system of equations).

To look for the traveling wave solution of (1), we use the transformation $u(x, t)=u(z), z=x-c t$ (where $c$ is a complex constant number). Then in (1), the system is carried to a system of ordinary differential equation:

$$
\begin{gathered}
-c u^{\prime}+\left(v^{2}\right)^{\prime}=0, \\
c v^{\prime}+a v^{\prime \prime \prime}-3 d v u^{\prime}-3 k u v^{2}=0 .
\end{gathered}
$$

By integrating the first equation in the system and neglecting the constant of integration we find

$$
c u=v^{2} .
$$

By inserting (4) into (3) and integration we find

$$
c^{2} v-(2 d+k) v^{3}+a c v^{\prime \prime}=0 .
$$

In 2007, El-Wakil and Abdou [5] got two solutions of (5) via tanh-function method for finding exact solutions of (5).

In this paper, we employ the complex method which was introduced by Yuan et al. [6-8] to obtain the general solutions and some new solutions of (5). In order to state our results, we need some concepts and notations.

A meromorphic function $w(z)$ means that $w(z)$ is holomorphic in the complex plane $\mathbb{C}$ except for poles. $\wp\left(z ; g_{2}, g_{3}\right)$ 
is the Weierstrass elliptic function with invariants $g_{2}$ and $g_{3}$. We say that a meromorphic function $f$ belongs to the class $W$ if $f$ is an elliptic function, or a rational function of $e^{\alpha z}$, $\alpha \in \mathbb{C}$, or a rational function of $z$.

Our main result is the following theorem.

Theorem 1. All meromorphic solutions $u$ of (5) belong to the class W. Furthermore, (5) has the following two forms of solutions:

(i) the elliptic general solutions

$$
\begin{aligned}
v_{d}(z) & \\
= \pm & \sqrt{\frac{a c}{4 d+2 k}} \\
\times & \left((-\wp+A)\left(4 \wp A^{2}+4 \wp^{2} A+2 \wp^{\prime} B-\wp g_{2}-A g_{2}\right)\right) \\
\times & \left(\left(\left(12 A^{2}-g_{2}\right) \wp+4 A^{3}-3 A g_{2}\right) \wp^{\prime}\right. \\
& \left.+4 B \wp^{3}+12 A B \wp^{2}-3 B g_{2} \wp-A B g_{2}\right)^{-1}
\end{aligned}
$$

where $g_{3}=0, B^{2}=4 A^{3}-g_{2} A, g_{2}$ and $A$ are arbitrary constants;

(ii) the simply periodic solutions, where $\xi=e^{\alpha z}$ are obtained, for $z_{0} \in \mathbb{C}$, by

$$
v_{s, 1}(\xi)= \pm c \frac{1}{\sqrt{2 d+k}}\left(\operatorname{coth} \frac{\alpha}{2}\left(z-z_{0}\right)\right)
$$

where $a=2 c / \alpha^{2}$

$$
v_{s, 2}(\xi)= \pm \sqrt{2} c \sqrt{\frac{-1}{2 d+k}} \frac{1}{\sinh \alpha\left(z-z_{0}\right)},
$$

where $a=-c / \alpha^{2}$;

$$
v_{s, 3}(\xi)= \pm c \sqrt{\frac{1}{2 d+k}}\left(\frac{1}{\sinh \alpha\left(z-z_{0}\right)}+1\right),
$$

where $a=c / 2 \alpha^{2}$.

Remark 2. Compared with the results of Wazwaz [4], we find that $v_{s, 3}(z)$ is the new solution of this Drinfeld-Sokolov equations (DS system of equations).

\section{Preliminary Lemmas and the Complex Method}

In order to give complex method and the proof of Theorem 1, we need some notations and results.

Set $m \in \mathbb{N}:=\{1,2,3, \ldots\}, r_{j} \in \mathbb{N}_{0}=\mathbb{N} \cup\{0\}, r=$ $\left(r_{0}, r_{1}, \ldots, r_{m}\right)$, and $j=0,1, \ldots, m$. We define a differential monomial denoted by

$$
M_{r}[w](z):=[w(z)]^{r_{0}}\left[w^{\prime}(z)\right]^{r_{1}}\left[w^{\prime \prime}(z)\right]^{r_{2}} \cdots\left[w^{(m)}(z)\right]^{r_{m}} .
$$

$p(r):=r_{0}+2 r_{1}+\cdots+(m+1) r_{m}$ and $\operatorname{deg}(M)$ are called the weight and degree of $M_{r}[w]$, respectively.

A differential polynomial $P\left(w, w^{\prime}, \ldots, w^{(m)}\right)$ is defined as follows:

$$
P\left(w, w^{\prime}, \ldots, w^{(m)}\right):=\sum_{r \in I} a_{r} M_{r}[w]
$$

where $a_{r}$ are complex constants and $I$ is a finite index set. The total weight and degree of $P\left(w, w^{\prime}, \ldots, w^{(m)}\right)$ are defined by $W(P):=\max _{r \in I}\{p(r)\}$ and $\operatorname{deg}(P):=\max _{r \in I}\left\{\operatorname{deg}\left(M_{r}\right)\right\}$, respectively.

We will consider the following complex ordinary differential equations:

$$
P\left(w, w^{\prime}, \ldots, w^{(m)}\right)=b w^{n}+c,
$$

where $b \neq 0, c$ are complex constants, $n \in \mathbb{N}$.

Let $p, q \in \mathbb{N}$. Suppose that (12) has a meromorphic solution $w$ with at least one pole; we say that (12) satisfies weak $\langle p, q\rangle$ condition if it substitutes Laurent series

$$
w(z)=\sum_{k=-q}^{\infty} c_{k} z^{k}, \quad q>0, c_{-q} \neq 0
$$

into (12). We can determine $p$ distinct Laurent singular parts as follows:

$$
\sum_{k=-q}^{-1} c_{k} z^{k}
$$

Lemma 3 (see [6-8]). Let $p, l, m, n \in \mathbb{N}$, $\operatorname{deg} P\left(w, w^{(m)}\right)<n$. Suppose that an $m$-order Briot-Bouquet equation

$$
P\left(w^{(m)}, w\right)=b w^{n}+c
$$

satisfies weak $\langle p, q\rangle$ condition; then all meromorphic solutions of (15) belong to the class $W$. If for some values of parameters such solution $w$ exists, then other meromorphic solutions form a one-parametric family $w\left(z-z_{0}\right), z_{0} \in \mathbb{C}$. Furthermore, each elliptic solution with pole at $z=0$ can be written as

$$
\begin{aligned}
& w(z) \\
& =\sum_{i=1}^{l-1} \sum_{j=2}^{q_{i}} \frac{(-1)^{j} c_{-i j}}{(j-1) !} \frac{d^{j-2}}{d z^{j-2}}\left(\frac{1}{4}\left[\frac{\wp^{\prime}(z)+B_{i}}{\wp(z)-A_{i}}\right]^{2}-\wp(z)\right) \\
& \quad+\sum_{i=1}^{l-1} \frac{c_{-i 1}}{2} \frac{\wp^{\prime}(z)+B_{i}}{\wp(z)-A_{i}}+\sum_{j=2}^{q_{l}} \frac{(-1)^{j} c_{-l j}}{(j-1) !} \frac{d^{j-2}}{d z^{j-2}} \wp(z)+c_{0},
\end{aligned}
$$

where $c_{-i j}$ are given by (13), $B_{i}^{2}=4 A_{i}^{3}-g_{2} A_{i}-g_{3}$, and $\sum_{i=1}^{l} c_{-i 1}=0$.

Each rational function solution $w:=R(z)$ is of the form

$$
R(z)=\sum_{i=1}^{l} \sum_{j=1}^{q} \frac{c_{i j}}{\left(z-z_{i}\right)^{j}}+c_{0}
$$

with $l(\leq p)$ distinct poles of multiplicity $q$. 
Each simply periodic solution is a rational function $R(\xi)$ of $\xi=e^{\alpha z}(\alpha \in \mathbb{C})$. $R(\xi)$ has $l(\leq p)$ distinct poles of multiplicity $q$ and is of the form

$$
R(\xi)=\sum_{i=1}^{l} \sum_{j=1}^{q} \frac{c_{i j}}{\left(\xi-\xi_{i}\right)^{j}}+c_{0} .
$$

In order to give the representations of elliptic solutions, we need some notations and results concerning elliptic function [9].

Let $\omega_{1}, \omega_{2}$ be two given complex numbers such that $\operatorname{Im}\left(\omega_{1} / \omega_{2}\right)>0$, and let $L=L\left[2 \omega_{1}, 2 \omega_{2}\right]$ be a discrete subset $L\left[2 \omega_{1}, 2 \omega_{2}\right]=\left\{\omega \mid \omega=2 n \omega_{1}+2 m \omega_{2}, n, m \in \mathbb{Z}\right\}$, which is isomorphic to $\mathbb{Z} \times \mathbb{Z}$. The discriminant $\Delta=\Delta\left(c_{1}, c_{2}\right):=$ $c_{1}^{3}-27 c_{2}^{2}$ and

$$
s_{n}=s_{n}(L):=\sum_{\omega \in L \backslash\{0\}} \frac{1}{\omega^{n}} .
$$

Weierstrass elliptic function $\wp(z):=\wp\left(z, g_{2}, g_{3}\right)$ is a meromorphic function with double periods $2 \omega_{1}, 2 \omega_{2}$ and satisfying the equation

$$
\left(\wp^{\prime}(z)\right)^{2}=4 \wp(z)^{3}-g_{2} \wp(z)-g_{3},
$$

where $g_{2}=60 s_{4}, g_{3}=140 s_{6}$, and $\Delta\left(g_{2}, g_{3}\right) \neq 0$.

If we changed (18) to the form

$$
\left(\wp^{\prime}(z)\right)^{2}=4\left(\wp(z)-e_{1}\right)\left(\wp(z)-e_{2}\right)\left(\wp(z)-e_{2}\right),
$$

we will have $e_{1}=\wp\left(\omega_{1}\right), e_{2}=\wp\left(\omega_{2}\right)$, and $e_{3}=\wp\left(\omega_{1}+\omega_{2}\right)$.

Inversely, given two complex numbers $g_{2}$ and $g_{3}$ such that $\Delta\left(g_{2}, g_{3}\right) \neq 0$, then there exists double periods $2 \omega_{1}, 2 \omega_{2}$ Weierstrass elliptic function $\wp(z)$ such that the previous results hold.

Lemma 4 (see $[9,10])$. Weierstrass elliptic functions $\wp(z):=$ $\wp\left(z, g_{2}, g_{3}\right)$ have two successive degeneracies and addition formula:

(i) degeneracy to simply periodic functions (i.e., rational functions of one exponential $e^{k z}$ ) according to

$$
\wp\left(z, 3 d^{2},-d^{3}\right)=2 d-\frac{3 d}{2} \operatorname{coth}^{2} \sqrt{\frac{3 d}{2}} z,
$$

if one root $e_{j}$ is double $\left(\Delta\left(g_{2}, g_{3}\right)=0\right)$;

(ii) degeneracy to rational functions of $z$ according to

$$
\wp(z, 0,0)=\frac{1}{z^{2}}
$$

if one root $e_{j}$ is triple $\left(g_{2}=g_{3}=0\right)$;

(iii) addition formula

$\wp\left(z-z_{0}\right)=-\wp(z)-\wp\left(z_{0}\right)+\frac{1}{4}\left[\frac{\wp^{\prime}(z)+\wp^{\prime}\left(z_{0}\right)}{\wp(z)-\wp\left(z_{0}\right)}\right]^{2}$.
By the previous lemma and results, we can give a new method next say complex method, to find exact solutions of some PDEs.

Step 1. Substituting the transform $T: u(x, t) \rightarrow$ $w(z),(x, t) \rightarrow z$ into a given PDE gives a nonlinear ordinary differential equations (12) or (15).

Step 2. Substitute (13) into (12) or (15) to determine that weak $\langle p, q\rangle$ condition holds.

Step 3. By determinant relation (16)-(18), we find the elliptic rational and simply periodic solutions $u(z)$ of (12) or (15) with pole at $z=0$, respectively.

Step 4. By Lemmas 3 and 4 , we obtain the all meromorphic solutions $w\left(z-z_{0}\right)$.

Step 5. By substituting the inverse transform $T^{-1}$ into these meromorphic solutions $w\left(z-z_{0}\right)$, we get all the exact solutions $u(x, t)$ of the original given PDE.

\section{Proof of Theorem 1}

By substituting (13) into (5), we have $q=1 . p=2, c_{-1}=$ $\pm \sqrt{2 a c /(2 d+k)}, c_{0}=0, c_{1}=\sqrt{2} c^{2} / 6 \sqrt{a c(2 d+k)}$, and $c_{2}=0$. Hence, (5) satisfies weak $\langle 2,1\rangle$ condition and is a 2 -order Briot-Bouquet differential equation. Obviously, (5) satisfies the dominant condition. So, by Lemma 3 , we know that all meromorphic solutions of (5) belong to $W$. Now, we will give the forms of all meromorphic solutions of (5).

By (16), we infer the indeterminate rational solutions of (5) with pole at $z=0$ that

$$
v_{r}(z)=\frac{c_{11}}{z}+\frac{c_{12}}{z-z_{1}}+c_{10} .
$$

By substituting $v_{r}(z)$ into (5), we get two distinct forms as follows:

$$
\begin{gathered}
v_{r, 1}(z)=0, \\
v_{r, 2}(z)= \pm c \sqrt{\frac{1}{2 d+k}} .
\end{gathered}
$$

We omit the constant solutions, and we obtain that there does not exist rational function solution.

In order to have simply periodic solutions, set $\xi=$ $\exp (\alpha z)$ and put $v=v(\xi)$ into (5), then

$$
a c \alpha^{2}\left(\xi^{2} v^{\prime \prime}+\xi v^{\prime}\right)-(2 d+k) v^{3}+c^{2} v=0
$$

By substituting

$$
v_{2}(\xi)=\frac{c_{2}}{\xi-1}+\frac{c_{1}}{\xi-\xi_{1}}+c_{0}
$$

into (5), we obtain the indeterminate simply periodic solutions of (27) with pole at $\xi=1$ that

$$
v_{s 1,1}(\xi)= \pm c \sqrt{\frac{1}{2 d+k}}\left(\frac{2}{\xi-1}+1\right),
$$

where $a=2 c / \alpha^{2}$; 


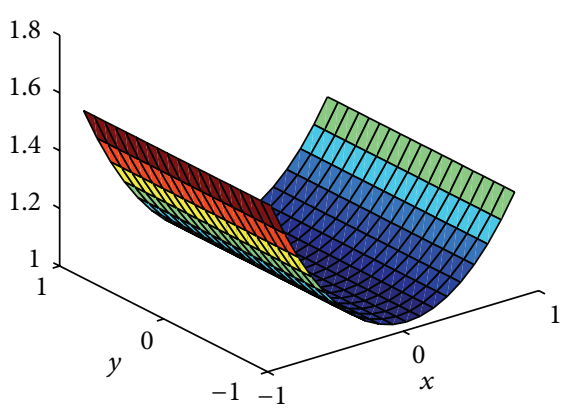

(a)

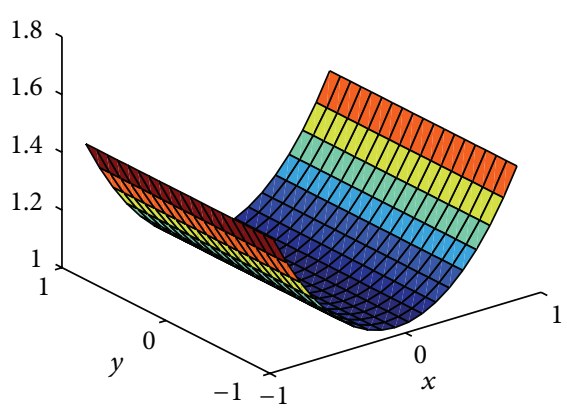

(b)

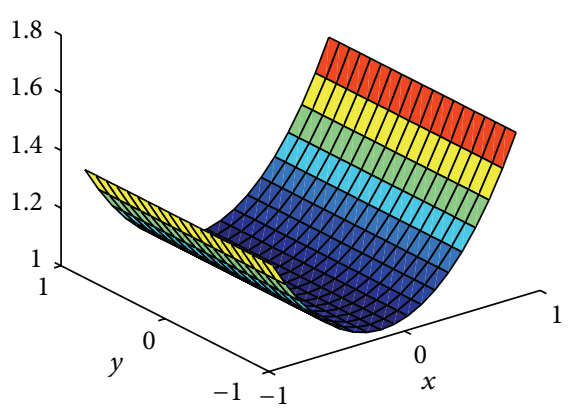

(c)

FIGURE 1: The solution of (DS) corresponding to $v_{s, 1}$ : take $c=1, z_{0}=0, d=1 / 2, k=0, \alpha=2$, (a) $t=-1 / 10$, (b) $t=0$, and (c) $t=1 / 10$.

$$
v_{s 1,2}(\xi)= \pm \sqrt{2} c \sqrt{\frac{-1}{2 d+k}}\left(\frac{1}{\xi-1}+\frac{1}{\xi+1}\right),
$$

where $a=-c / \alpha^{2}$;

$$
v_{s 1,3}(\xi)= \pm c \sqrt{\frac{1}{2 d+k}}\left(\frac{1}{\xi-1}+\frac{1}{\xi+1}+1\right),
$$

where $a=c / 2 \alpha^{2}$.

By substituting $\xi=e^{\alpha z}$ into the previous six relations, we get all simply periodic solutions of (5) with pole at $z=0$ :

$$
v_{s 0,1}(z)= \pm c \sqrt{\frac{1}{2 d+k}}\left(\frac{2}{e^{\alpha z}-1}+1\right),
$$

where $a=2 c / \alpha^{2}$;

$$
v_{s 0,2}(z)= \pm \sqrt{2} c \sqrt{\frac{-1}{2 d+k}}\left(\frac{1}{e^{\alpha z}-1}+\frac{1}{e^{\alpha z}+1}\right),
$$

where $a=-c / \alpha^{2}$;

$$
v_{s 0,3}(z)= \pm c \sqrt{\frac{1}{2 d+k}}\left(\frac{1}{e^{\alpha z}-1}+\frac{1}{e^{\alpha z}+1}+1\right),
$$

where $a=c / 2 \alpha^{2}$.

So all simply periodic solutions of (5) are obtained, for $z_{0} \in \mathbb{C}$, by

$$
\begin{aligned}
v_{s, 1}(z) & = \pm c \sqrt{\frac{1}{2 d+k}}\left(\frac{2}{e^{\alpha\left(z-z_{0}\right)}-1}+1\right) \\
& = \pm c \frac{1}{\sqrt{2 d+k}}\left(\operatorname{coth} \frac{\alpha}{2}\left(z-z_{0}\right)\right),
\end{aligned}
$$

where $a=2 c / \alpha^{2}$;

$$
\begin{aligned}
v_{s, 2}(z) & = \pm \sqrt{2} c \sqrt{\frac{-1}{2 d+k}}\left(\frac{1}{e^{\alpha\left(z-z_{0}\right)}-1}+\frac{1}{e^{\alpha\left(z-z_{0}\right)}+1}\right) \\
& = \pm \sqrt{2} c \sqrt{\frac{-1}{2 d+k}} \frac{1}{\sinh \alpha\left(z-z_{0}\right)},
\end{aligned}
$$

where $a=-c / \alpha^{2}$;

$$
\begin{aligned}
v_{s, 3}(z) & = \pm c \sqrt{\frac{1}{2 d+k}}\left(\frac{1}{e^{\alpha\left(z-z_{0}\right)}-1}+\frac{1}{e^{\alpha\left(z-z_{0}\right)}+1}+1\right) \\
& = \pm c \sqrt{\frac{1}{2 d+k}}\left(\frac{1}{\sinh \alpha\left(z-z_{0}\right)}+1\right),
\end{aligned}
$$

where $a=c / 2 \alpha^{2}$.
From (15) in Lemma 3, we have indeterminant relations of elliptic solutions of (5) with pole at $z=0$ :

$$
v_{d 0}(z)= \pm \sqrt{\frac{a c}{4 d+2 k}} \frac{\wp^{\prime}(z)+F}{\wp(z)-E}+c_{30},
$$

where $F^{2}=4 E^{3}-g_{2} E-g_{3}$. By applying the conclusion (ii) of Lemma 4 to $u_{d 0}(z)$ and noting that the results of rational solutions obtained previously, we deduce that $c_{30}=0, E=$ $F=0$, and $g_{3}=0$. Then, we get that

$$
v_{d 0}(z)= \pm \sqrt{\frac{a c}{4 d+2 k}} \frac{\wp^{\prime}(z)}{\wp(z)} .
$$
follows

Therefore, all elliptic function solutions of (5) are as

$$
v_{d 0}(z)= \pm \sqrt{\frac{a c}{4 d+2 k}} \frac{\wp^{\prime}\left(z-z_{0}\right)}{\wp\left(z-z_{0}\right)} .
$$

Here $z_{0} \in \mathbb{C}, g_{3}=0$. Making use of the addition of Lemma 3 we rewrite it to the form

$$
\begin{aligned}
& v_{d}(z) \\
& = \pm \sqrt{\frac{a c}{4 d+2 k}} \\
& \times\left((-\wp+A)\left(4 \wp A^{2}+4 \wp^{2} A+2 \wp^{\prime} B-\wp g_{2}-A g_{2}\right)\right) \\
& \times\left(\left(\left(12 A^{2}-g_{2}\right) \wp+4 A^{3}-3 A g_{2}\right) \wp^{\prime}\right. \\
& \left.\quad+4 B \wp^{3}+12 A B \wp^{2}-3 B g_{2} \wp-A B g_{2}\right)^{-1} .
\end{aligned}
$$

Here $g_{3}=0, B^{2}=4 A^{3}-g_{2} A, g_{2}$, and $A$ are arbitrary constants.

This completes the proof of Theorem 1.

\section{Computer Graphs for New Solutions}

In this section, we give some computer graphs to illustrate our main results. Here, we take the simple periodic solutions $v_{s, 1-3}(z)$ by Figures 1,2 , and 3 . 


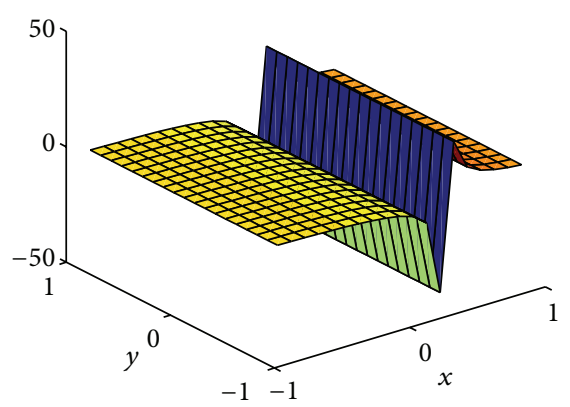

(a)

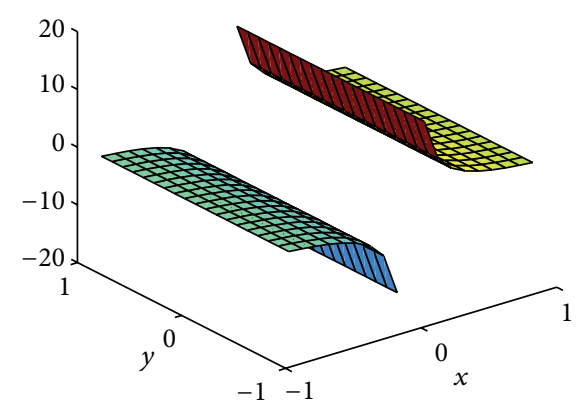

(b)

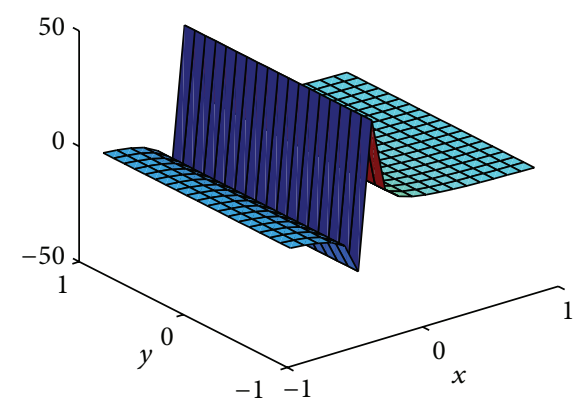

(c)

FIgURE 2: The solution of (DS) corresponding to $v_{s, 2}$ : take $c=1, z_{0}=0, d=-1 / 2, k=0, \alpha=1$, (a) $t=-1 / 3$, (b) $t=0$, and (c) $t=1 / 3$.

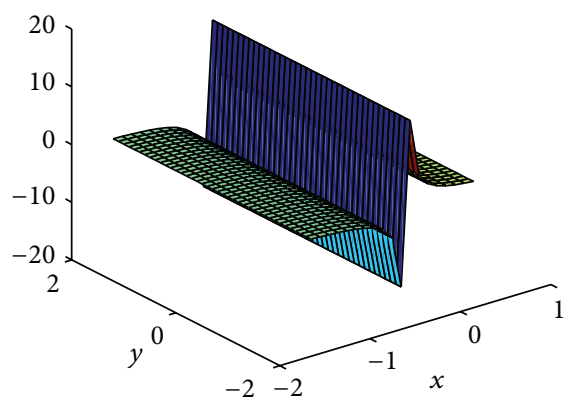

(a)

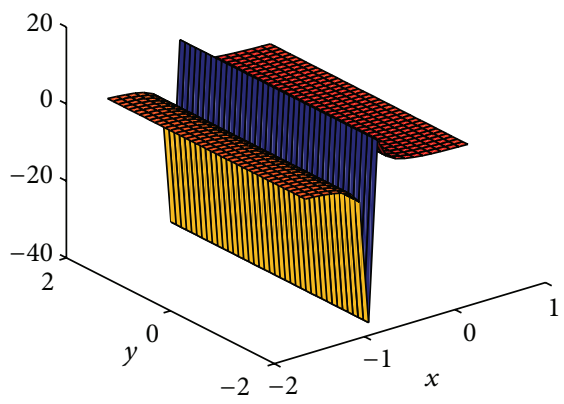

(b)

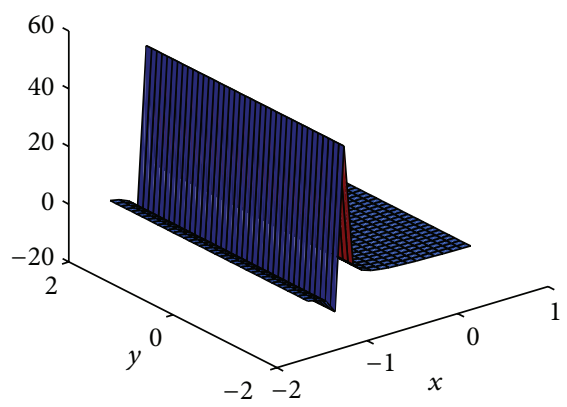

(c)

FIGURE 3: The solution of (DS) corresponding to $v_{s, 3}$ : take $c=1, z_{0}=0, d=1 / 2, k=0, \alpha=1$, (a) $t=-1 / 3$, (b) $t=0$, and (c) $t=1 / 3$.

\section{Conclusions}

Complex method is a very important tool in finding the exact solutions of nonlinear evolution equations, and the DrinfeldSokolov equations (DS system of equations) are a classic and simplest case of the nonlinear reaction-diffusion equation. In this paper, we employ the complex method to obtain the general meromorphic solutions of the Drinfeld-Sokolov equations (DS system of equations), which improves the corresponding result obtained by El-Wakil and Abdou, [5]. Our results show that simply periodic traveling wave exact solutions of the equations (DS) are solitary wave solutions, the complex method is simpler than other methods and there does not exist any rational solutions and there exist simply periodic solutions $v_{s, 3}(z)$ which are not only new but also not degenerated successively by the elliptic function solutions. We believe that this method should play an important role in finding exact solutions in the mathematical physics. For these new traveling wave solutions, we give some computer simulations to illustrate our main results.

\section{Acknowledgments}

This work was supported by the Visiting Scholar Program of Chern Institute of Mathematics at Nankai University where the second and third authors worked as visiting scholars. This work was supported by the NNSF of China (nos. 11271090, 11171184, and 11001057), the NSF of Guangdong Province (S2012010010121), and Shanghai University Young Teacher
Training Program (ZZSDJ12020) and supported by projects 10XKJ01, 12C401, and 12C104 from the Leading Academic Discipline Project of Shanghai Dianji University.

\section{References}

[1] Ü. Göktaş and W. Hereman, "Symbolic computation of conserved densities for systems of nonlinear evolution equations," Journal of Symbolic Computation, vol. 24, no. 5, pp. 591-621, 1997.

[2] P. J. Olver, Applications of Lie Groups to Differential Equations, vol. 107 of Graduate Texts in Mathematics, Springer, New York, NY, USA, 2nd edition, 1993.

[3] J. P. Wang, "A list of $1+1$ dimensional integrable equations and their properties," Journal of Nonlinear Mathematical Physics, vol. 9, no. suppl. 1, pp. 213-233, 2002.

[4] A.-M. Wazwaz, "Exact and explicit travelling wave solutions for the nonlinear Drinfeld-Sokolov system," Communications in Nonlinear Science and Numerical Simulation, vol. 11, no. 3, pp. 311-325, 2006.

[5] S. A. El-Wakil and M. A. Abdou, "Modified extended tanhfunction method for solving nonlinear partial differential equations," Chaos, Solitons \& Fractals, vol. 31, no. 5, pp. 1256-1264, 2007.

[6] W. J. Yuan, Y. Z. Li, and J. M. Lin, "Meromorphic solutions of an auxiliary ordinary differential equation using complex method," Mathematical Methods in the Applied Sciences, vol. 36, no. 13, pp. 1776-1782, 2013.

[7] W. Yuan, Y. Huang, and Y. Shang, "All traveling wave exact solutions of two nonlinear physical models," Applied Mathematics and Computation, vol. 219, no. 11, pp. 6212-6223, 2013. 
[8] W. J. Yuan, Y. D. Shang, Y. Huang, and H. Wang, “The representation of meromorphic solutions of certain ordinary differential equations and its applications," Scientia Sinica Mathematica, vol. 43, no. 7, 2013.

[9] S. Lang, Elliptic Functions, vol. 112 of Graduate Texts in Mathematics, Springer, New York, NY, USA, 2nd edition, 1987.

[10] R. Conte and M. Musette, "Elliptic general analytic solutions," Studies in Applied Mathematics, vol. 123, no. 1, pp. 63-81, 2009. 


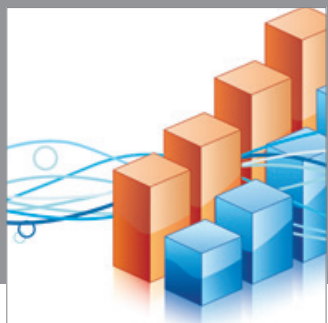

Advances in

Operations Research

mansans

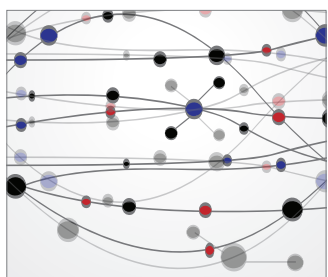

The Scientific World Journal
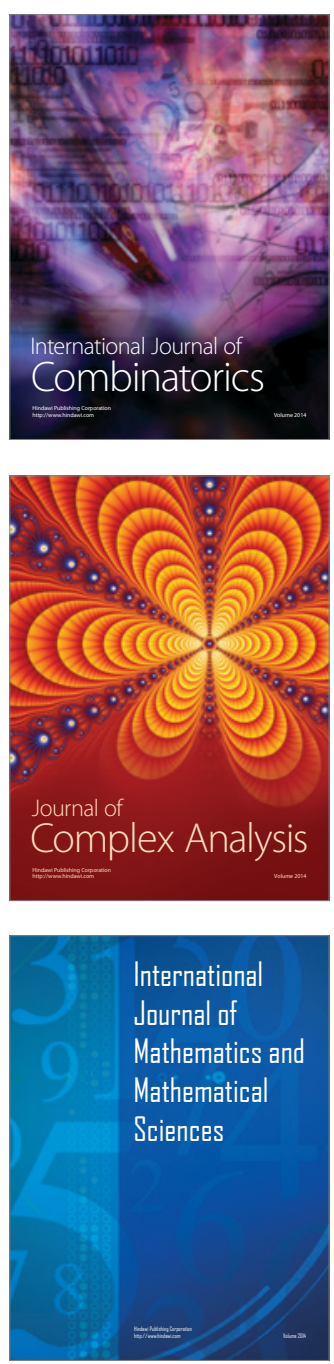
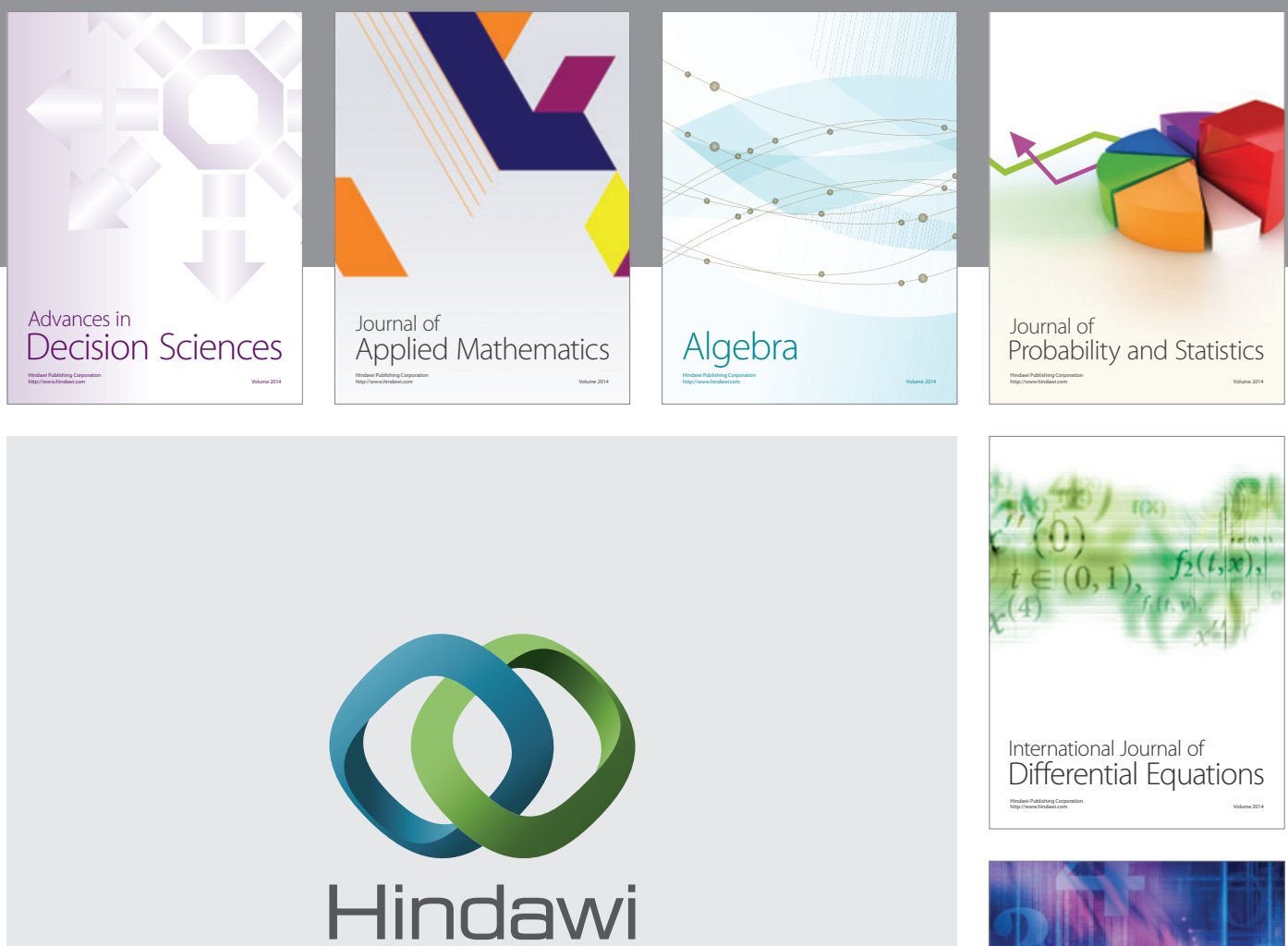

Submit your manuscripts at http://www.hindawi.com
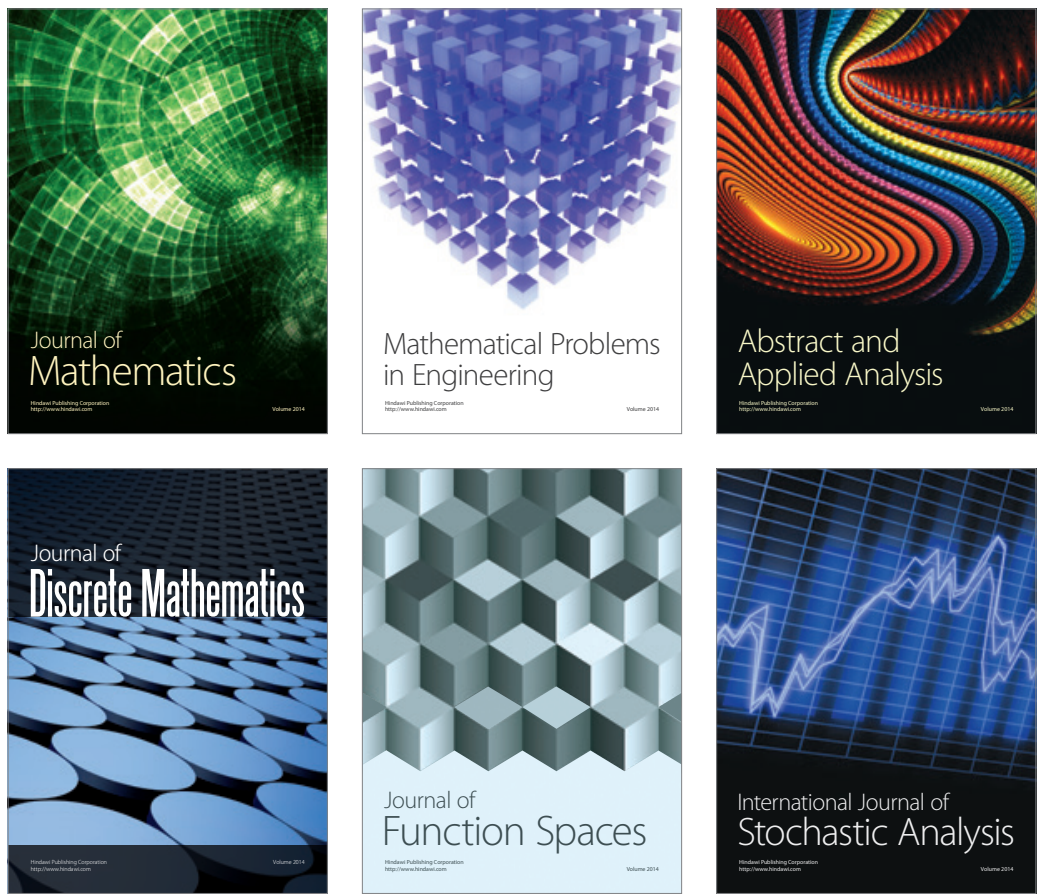

Journal of

Function Spaces

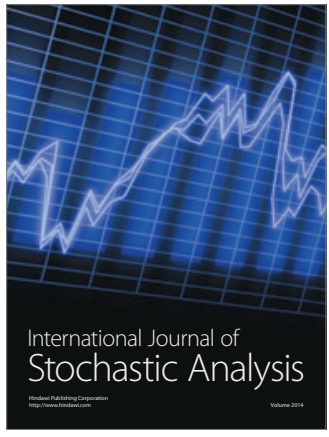

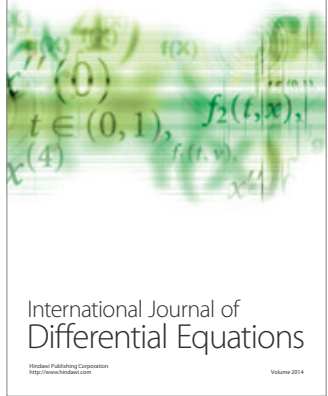
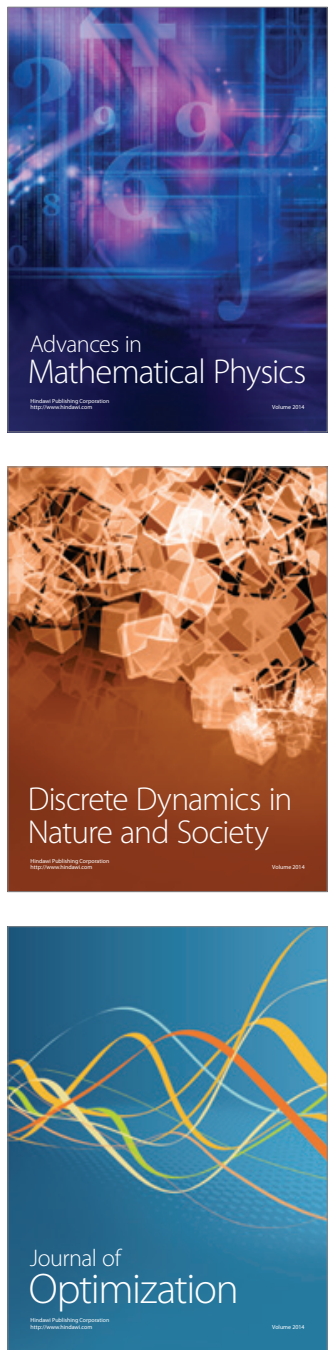